\title{
Mass Spectrum of exotic Hyperatoms
}

\section{Arezu Jahanshir}

Department of Physics and Engineering Sciences, Imam Khomeini International University, Buein Zahra Higher Education Centre of Engineering and Technology, Iran.

\begin{tabular}{|c|c|}
\hline ARTICLE INFO & ABSTRACT \\
\hline $\begin{array}{l}\text { Article history: } \\
\text { Received: } 27^{\text {th }} \text { June } 2021 \\
\text { Accepted: } 3^{\text {rd }} \text { Oct. } 2021 .\end{array}$ & $\begin{array}{l}\text { The hyperatoms belong to the group of exotic atoms. An exotic hyperatom is a system } \\
\text { where a negative muon as an electron in a normal atom is captured into an } \\
\text { hyperatomic orbit around hypernuclei. Our knowledge of the non-relativistic }\end{array}$ \\
\hline $\begin{array}{l}\text { Keywords: } \\
\text { Hypernuclei, } \\
\text { Schrödinger equation, } \\
\text { Hyperatoms, }\end{array}$ & $\begin{array}{l}\text { quantum mechanics is a good method for the exotic hyperatoms containing one } \\
\text { muon. We theoretically have been investigated exotic muonic hyperatoms. } \\
\text { Theoretically, the mass spectra of muonic hyperatoms have been determined in the } \\
\text { framework of the non-relativistic Schrödinger equation with Coulomb type potential }\end{array}$ \\
\hline Exotic atoms. & $\begin{array}{l}\text { describing the interaction between a muon and the hypernuclei core. The studies of } \\
\text { exotic systems have achieved great progress both in theory and experiments. Mass } \\
\text { spectrum and binding energy have been provided useful information for exotic } \\
\text { hadronic systems and for hypernuclei which participate in the experimental reaction } \\
\text { and also the interaction between them. The analytic method based on quantum field } \\
\text { theories, the behavior of the correlation function, Feynman path integral, and } \\
\text { oscillator representation method is suggested. Within all of these different } \\
\text { frameworks, we have presented the theoretical approach to describing the mass } \\
\text { spectrum of muonic hyperatoms in the ground and orbital excited states. }\end{array}$ \\
\hline
\end{tabular}

\section{INTRODUCTION}

Determination of hyperatoms masses by exact mass formula has been a very important issue in theoretical nuclear physics. This article proposes a well-known method called the oscillator representation method (ORM), which determines the bound state mass and constituent masses of hypernuclei and muon. The mass spectrum of the exotic atom as a bound state can be determined within the quantum field theory due to particle charge. The scalar charged particles current is $J(x)=\Psi^{+}(x) \Psi^{-}(x)$, for defining the bound state mass based on correlator $\Pi(x)$ which can be described by Green's function $G_{m}\left(x_{i}, x_{j} \mid E(x)\right)$, all annihilation channels are neglected and Green's function product of scalar particles with masses $m_{1}, m_{2}$ in the external electrostatic field will be given as correlator $\Pi(x)_{\left|x=x_{1}-x_{2}\right|}=\left\langle G_{m_{1}}\left(x_{1}, x_{2} \mid E\right) \cdot G_{m_{2}}^{*}\left(x_{2}, x_{1} \mid E\right)\right\rangle$. The

Green's function is presented in the functional integral form [2-4] $(\hbar=c=1)$

$$
\begin{aligned}
& \left\langle J\left(x_{1}\right) J\left(x_{2}\right)\right\rangle_{E(x)}=\left\langle\Psi^{+}\left(x_{1}\right) \Psi^{-}\left(x_{2}\right) \Psi^{+}\left(x_{2}\right) \Psi^{-}\left(x_{1}\right)\right\rangle_{E(x)} \\
& \left\langle\Psi\left(x_{1}\right) \Psi\left(x_{2}\right)\right\rangle_{E(x)}=\int \frac{d k}{2 \pi} \widetilde{D}\left(k^{2}\right) e^{-i k\left(x_{1}-x_{2}\right)} \\
& G_{m}\left(x_{1}, x_{2} \mid E(x)\right) \\
& =\int_{0}^{\infty} \frac{d u}{(4 \pi u)^{2}} e^{-u m^{2}-\frac{x^{2}}{4 u}} \times \int N B \delta(x) e^{-0.5 \int_{0}^{1} \dot{B}^{2}(\eta) d \eta+i g \int_{0}^{s} A(\eta) \frac{\partial Z(\eta)}{\partial \eta} d \eta}
\end{aligned}
$$

In quantum field theory the bound state mass is defined with this equation $\Pi(x)_{\left|x=x_{1}-x_{2}\right| \rightarrow \infty} \approx e^{-M|x|}$, then after averaging over the external electrostatic field $E(x)$ one also finds a correlator on functional integral form [1] 


$$
\begin{aligned}
& \Pi(x)_{|x| \rightarrow \infty} \\
& =\int_{0}^{\infty} \int_{0}^{\infty} \frac{d \mu_{1} d \mu_{2}}{\left(8(x) \pi^{2}\right)^{2}} J\left(\mu_{1}, \mu_{2}\right) e^{-\frac{|x|}{2}\left(\frac{m_{1}^{2}}{\mu_{1}}+\frac{m_{2}^{2}}{\mu_{2}}+\mu_{1}+\mu_{2}\right)}
\end{aligned}
$$

$J\left(\mu_{1}, \mu_{2}\right)$-contains potential and nonpotential interactions. This article will not focus on relativistic correction for potential interactions, because the aim is to determine the mass spectrum and the related mass of the system. But in the asymptotic limit $[1,3]$

$\lim _{|x| \rightarrow \infty} J\left(\mu_{1}, \mu_{2}\right)=e^{-(x) E_{\ell}\left(\mu_{1}, \mu_{2}\right)}$

Where function $E_{\ell}\left(\mu_{1}, \mu_{2}\right)=E_{\ell}(\mu)$ is the eigenvalue of the interaction Hamiltonian:

$$
\begin{aligned}
& \hat{H} \Psi(x)=E_{\ell}\left(\mu_{1}, \mu_{2}\right) \Psi(x) \Rightarrow \hat{H}=\sqrt{\hat{p}^{2}+m_{1}^{2}}+ \\
& \sqrt{\hat{p}^{2}+m_{2}^{2}}+V(x)
\end{aligned}
$$

Then the bound state of hyperatom mass is defined as a minimum of relation

$M=\frac{1}{2} \min _{\mu_{1}, \mu_{2}}\left[\frac{m_{1}^{2}}{\mu_{1}}+\frac{m_{2}^{2}}{\mu_{2}}+\mu_{1}+\mu_{2}+2 E_{\ell}(\mu)\right]=$

$\sqrt{m_{1}^{2}-2 \mu^{2} E_{\ell}^{\prime}(\mu)}+\sqrt{m_{2}^{2}-2 \mu^{2} E_{\ell}^{\prime}(\mu)}+\mu E_{\ell}^{\prime}(\mu)+E_{\ell}(\mu)$

$\mu_{1}=\sqrt{m_{1}^{2}-2 \mu^{2} E_{\ell}^{\prime}(\mu)}, \quad \mu_{2}=\sqrt{m_{2}^{2}-2 \mu^{2} E_{\ell}^{\prime}(\mu)}, \quad E_{\ell}^{\prime}(\mu)=\frac{\partial E_{\ell}(\mu)}{\partial \mu}$

$\mu_{1}, \mu_{2}-$ are the constituent mass of hypernuclei and muon in the bound state. $m_{1}, m_{2}-$ are their rest mass, and $\mu=\frac{\mu_{1} \mu_{2}}{\mu_{1}+\mu_{2}}-$ is the bound state mass appearing in the n-body systems ( the reduced mass ). The binding energy for hyperatom like the normal atoms is calculated by (6) if the recoil effect is neglected i.e.:

$$
\begin{aligned}
& m_{1}=\infty, \\
& E_{\text {bin }}=M-m_{1}, \\
& E_{\text {bin }}=\sqrt{m_{2}^{2}-2 \mu^{2} E_{\ell}^{\prime}(\mu)}+\mu E_{\ell}^{\prime}(\mu)+E_{\ell}(\mu),
\end{aligned}
$$

\section{Hydrogen-muonic hyperatom}

The interdiction section presented the bound state mass of two body systems based on the asymptotic behavior of the correlation functions of the electrostatic field currents. In addition, it presented a theoretical method for determining the bound state mass of hydrogen-muonic hyperatoms consisting of ${ }_{\Lambda}^{x} H$ and $\mu^{-}$with relativistic mass correction [4]. The dependence of the constituent mass of hypernuclei and muon on the current mass is analytically derived. Now the parameters should be determined for the calculation of the masses. Therefore, based on basic elements of the oscillator representation method (ORM), the hydrogenmuonic hyperatom mass spectrum can be determined by applying the transformation into the new 4D momentum space [3]. In quantum field theory, for the ground and excited states, the systems are described by an infinite number of oscillators that keep their oscillating characteristic in the interactions. To describe the mechanism of interaction and creation of exotic hyperatom, the Schrödinger equation is generally invoked based on the Gaussian solution for large distances. Therefore, we are presented the radial Schrödinger equations that is applied for spherically symmetric Coulomb potential $\left(V(x)=\alpha_{S} x^{-1}\right)$ between the clusters (i.e., the hypernuclear core and the muon) and reads as follows[3]:

$\widehat{H} R(x)=E_{\ell}(\mu) R(x) \Rightarrow\left(\frac{\hat{p}^{2}}{2 \mu}+\alpha_{S} x^{-1}\right) R(x)=$
$E_{\ell}(\mu) R(x)$,

and to use quantum field methods of scalar field (the Gaussian solution), the variables $(x \rightarrow x(q))$ in the equation (7) have been changed by the following substitution:

$$
x=x(q)=q^{2} \Rightarrow \Phi_{\ell}(x) \rightarrow q^{2 \ell} \phi_{\ell}\left(q^{2}\right)
$$

and the radial Laplacian operator reads $\Delta=\frac{d^{2}}{d x^{2}}+$ $\frac{m-1}{x} \frac{d}{d x}$, and the Schrödinger equation (7) reads:

$$
\begin{aligned}
\left(-\frac{1}{2 x}\left(\frac{d}{d x}\right)^{2} x\right. & +\frac{\ell(\ell+1)}{2 x^{2}} \frac{d}{d x} \\
& +V(x)) R(x)=E_{\ell}(\mu) R(x)
\end{aligned}
$$

then the radial Laplacian operator in the new 4D momentum space is written in the following form

$$
\begin{aligned}
& \hat{p}_{x}^{2} \rightarrow \frac{1}{4 q^{2}} \hat{p}_{q}^{2} \\
& \nabla_{x}^{2} \rightarrow \nabla_{q}^{2}=\frac{d^{2}}{d q^{2}}+\frac{3+4 \ell}{q} \frac{d}{d q}
\end{aligned}
$$


after standard simplifications, we have obtained the modified radial Schrödinger equations, in the Coulombic potential where the wave function becomes an oscillator one. The modified Hamiltonian $\hat{H} \phi_{\ell}(q)=E_{\ell}(\mu) \phi_{\ell}(q)$ with Gaussian asymptotic of wave functions of hyperatom interactions has a start point to describe energy and masses. Based on ORM and $\left(\hat{a}^{+}, \hat{a}^{-}\right)$ operators, the canonical variables $\left(\hat{q}, \hat{p}_{q}\right)$ in the (10) should be rewritten as:

$$
\begin{array}{r}
\hat{a}^{+}=\sqrt{\frac{\omega}{2}\left(\hat{x}-\frac{i}{\omega} \hat{p}\right), \quad \hat{a}^{-}=\sqrt{\frac{\omega}{2}}\left(\hat{x}+\frac{i}{\omega} \hat{p}\right), \quad \hat{x}} \\
=\frac{\hat{a}^{-}+\hat{a}^{+}}{\sqrt{2 \omega}}, \quad \hat{p}=\sqrt{\frac{\omega}{2} \frac{\hat{a}^{-}-\hat{a}^{+}}{i}}
\end{array}
$$

and then it is necessary to extract the exact pure oscillator segment that has the frequency $\omega$ and rewrite Hamiltonian in the normal form of operators $\hat{a}^{+}, \hat{a}^{-}$ based on (10). In this way, we have defined

$$
q^{2}=\frac{2(1+\ell)}{\omega_{\ell}}, \quad p^{2}=2(1+\ell) \omega_{\ell}
$$

for more details see [3]. Then by definition, the free Hamiltonian of a quantum system $\left(\varepsilon_{0}(\omega)\right)$ that oscillates with $\omega$ in the Coulomb potential interactions is obtained the new 4D momentum space [3-7]

$$
\begin{aligned}
& \widehat{H}_{\ell} \phi_{\ell}(q)=E_{\ell}(\mu) \phi_{\ell}(q) \\
& \Rightarrow\left(\frac{\hat{p}_{q}^{2}}{2 \mu}+4 q^{2}\left(V(q)-E_{\ell}(\mu)\right)\right) \phi_{\ell}(q) \\
& =0 \Rightarrow \varepsilon_{0}(\omega) \phi_{\ell}(q)=0 \\
& \varepsilon_{0}(\omega)=(1+\ell) \omega+4 \mu \alpha_{s} q^{2}\left(-\frac{\alpha_{s}}{q^{2}}-E_{\ell}(\mu)\right)=0
\end{aligned}
$$

ORM has required that the interaction segment of the Hamiltonian be free of quadratic $\left(q^{2}\right)$ because by theory and based on essential constraints on ORM, this part lies in free oscillator segments. This condition provides an equation for free oscillator frequency $\omega_{\ell}$ (i.e. $\varepsilon_{0}(\omega)$ is free of quadratic terms $\left(q^{2}\right)$ )[3]. The oscillator frequency $\omega$, which determines the main quantum contribution in the next equations. Calculation of energy eigenvalue $E_{\ell}(\mu)$ is defined from ORM conditions with $H_{\ell}-$ Hamiltonian in the zeroth approximation [8]

$$
\begin{aligned}
\left(\varepsilon_{0}\left(\omega_{\ell}\right)=0, \quad \frac{\partial \varepsilon_{0}\left(\omega_{\ell}\right)}{\partial \omega_{\ell}}=0\right) & \\
& \Rightarrow\left(E_{\ell}(\mu)\right. \\
& =\frac{1}{8 \mu} \omega_{\ell}^{2}-\frac{\alpha_{s} \Gamma(2+2 \ell)}{\Gamma(3+2 \ell)} \omega_{\ell}, \omega_{\ell} \\
& \left.=\frac{4 \mu \alpha_{s} \Gamma(2+2 \ell)}{\Gamma(3+2 \ell)}\right)
\end{aligned}
$$

Then it will be possible to determine the mass spectrum of hyperatom in the ground state [8-11] and with orbital excitation, $\ell=1$.

\section{RESULTS AND DISCUSSION}

The ORM is a projective unitary representation of the symplectic group or oscillator explanation that we have determined parameters for the ground and excited states with the well-known potential interaction. The oscillator method is the representation of the canonical variables in the normal form ( also called Wick order) and in the auxiliary space. Taking into account this representation we have obtained the expression for the ground and excited energy for the interaction Hamiltonian. We have defined the approach for predicting the mass spectrum of exotic hyperatom, based totally on the relativisticquantum model. Two body exotic muonic hyperatom mass spectrum has been expressed as

$$
\begin{aligned}
& M=\sqrt{m_{\text {hypernuclei }}^{2}-2 \mu^{2} E_{\ell}^{\prime}(\mu)} \\
& +\sqrt{m_{\mu}^{2}-2 \mu^{2} E_{\ell}^{\prime}(\mu)}+\mu E_{\ell}^{\prime}(\mu) \\
& +E_{\ell}(\mu)
\end{aligned}
$$

and binding energy as a normal atom described by

$$
E_{\text {bin }}=M-m_{\text {hypernuclei }}
$$

In the ORM approach, we have calculated the mass spectra of the exotic muonic hydrogen hypernuclei-bound state. The results of numerical calculation of all parameters that have mass dimension are presented with different constant interactions from coulomb $\alpha_{s}=0.007$ to semi-strong interaction $\alpha_{s}=0.5$. The ground and 
excited states bound state mass of two body hyperatom systems are determined from equations (15) and (16). The numerical results are shown in Tables (1) and Tables (2). with the rest values of particles in hydrogen hyperatoms (all mass values are given in $(\mathrm{MeV})$ ):

$$
\begin{aligned}
& m_{n}=939.565421 \quad m_{p}=938.272088 \\
& m_{\Lambda}=1115.683, \quad m_{\mu}=105.658376 \\
& { }_{\Lambda}^{3} H=2993.521, \quad{ }_{\Lambda}^{4} H=3933.086, \quad{ }_{\Lambda}^{5} H=4872.651, \\
& { }_{\Lambda}^{6} H=5812.217, \quad{ }_{\Lambda}^{7} H=6751.782
\end{aligned}
$$

\begin{tabular}{|c|c|c|c|c|c|c|}
\hline$\alpha_{s}$ & ${ }_{\Lambda}^{3} H$ & ${ }_{\Lambda}^{4} H$ & ${ }_{\Lambda}^{5} H$ & ${ }_{\Lambda}^{6} H$ & ${ }_{\Lambda}^{7} H$ & $\frac{E_{b i n}}{m_{\mu}}\left({ }_{\Lambda}^{3} H\right)$ \\
\hline 0.007 & 3099.1776 & 4038.7430 & 4978.3084 & 5917.8739 & 6857.4393 & 0.9997 \\
\hline 0.1 & 3098.9144 & 4038.4798 & 4978.0452 & 5917.6107 & 6857.1761 & 0.9975 \\
\hline 0.2 & 3098.1170 & 4037.6824 & 4977.2478 & 5916.8132 & 6856.3786 & 0.9899 \\
\hline 0.3 & 3096.7742 & 4036.3396 & 4975.9050 & 5915.4705 & 6855.0359 & 0.9772 \\
\hline 0.4 & 3094.8645 & 4034.4299 & 4973.9953 & 5913.5607 & 6853.1261 & 0.9592 \\
\hline 0.5 & 3092.3549 & 4031.9203 & 4971.4857 & 5911.0511 & 6850.6166 & 0.9354 \\
\hline
\end{tabular}

\begin{tabular}{|c|c|c|c|c|c|c|}
\hline$\alpha_{s}$ & ${ }_{\Lambda}^{3} H$ & ${ }_{\Lambda}^{4} H$ & ${ }_{\Lambda}^{5} H$ & ${ }_{\Lambda}^{6} H$ & ${ }_{\Lambda}^{7} H$ & $\frac{E_{b i n}}{m_{\mu}}\left({ }_{\Lambda}^{3} H\right)$ \\
\hline 0.007 & 3099.1782 & 4038.7437 & 4978.3091 & 5917.8745 & 6857.4399 & 0.9999 \\
\hline 0.1 & 3099.0467 & 4038.6121 & 4978.1776 & 5917.7430 & 6857.3084 & 0.9994 \\
\hline 0.2 & 3098.6493 & 4038.2147 & 4977.7801 & 5917.3455 & 6856.9109 & 0.9975 \\
\hline 0.3 & 3097.9835 & 4037.5489 & 4977.1143 & 5916.6797 & 6856.2451 & 0.9944 \\
\hline 0.4 & 3097.0442 & 4036.6096 & 4976.1750 & 5915.7404 & 6855.3058 & 0.9899 \\
\hline 0.5 & 3095.8238 & 4035.3892 & 4974.9546 & 5914.5201 & 6854.0855 & 0.9843 \\
\hline
\end{tabular}

Table (1): Mass spectrum and binding energy of exotic muonic hypernuclei $l=0$ in $(\mathrm{MeV})$

Table (2): Mass spectrum and binding energy of exotic muonic hypernuclei $l=1$ in (MeV) 


\section{CONCLUSION}

The exotic hyperatoms parameters have previously been studied in the low/high energy interactions with several potential models and models like the Nijmegen model, soft-core model, hard-core model, multi mesons exchange model, effective field theory chiral model, quark model, etc. This paper is devoted to determining theoretically the mass and binding energy in the framework of the ORM. An analytic expression for masses of exotic hyper systems, taking into account relativistic corrections. Without spin, interactions are derived. We have computed the masses of hyperatoms by considering the system as the nuclear core and muon. The inter hadronic interaction has been taken as electrostatically one boson exchange model. Our results have been taken from the theoretical ORM, are compared and identified with some of the experimentally or other theoretical data [7-11]. Some of our predictions bound states shown in Table1and 2 are presented as an exotic hadronic state. Maybe some of the new exotic hypernuclei-bound states very soon could be experimentally discovered in the new generation facilities, such as J-PARC, MAMI, JLab, and FAIR.

\section{ACKNOWLEDGEMENT}

This research was supported by the department of physics and engineering sciences, Buein Zahra Technical University.

\section{REFERENCES}

[1] Greiner, W., and Reinhort, Y., (1992) Quantum Electrodynamic, $1^{\text {st }}$ edition, Springer- Verleg.
[2] Rosenfelder, R., (2012) Path Integrals in Quantumt Physics, arXiv:1209.1315v2 [nuclth].

[3] Dineykhan, M., et al., (1995) Oscillator Representation in Quantum Physics, SpringerVerlag Berlin Heidelberg.

[4] Thakkar, K.et al., (2011) P-wave $\Lambda$-hyperon binding energies of medium-heavy Hypernuclei, Proc. DAE Symp. Nucl. Phys. 56, 750- 764 .

[5] Juric, M., (1973) Determination of the bindingenergy values of the light hypernuclei $(\mathrm{A}<15)$, Nuclear physics B2, 1-30.

[6] Crayton, N., et al., (1962) Compilation of Hyper Fragment Binding Energies, Reviews of Modern Physics, 34(2), 186-189.

[7] Dover, C., Gal, A., (1983), Hypernuclei, Annals of Physics, 146 (2), 309-348.

[8] Pal, S., (2017) A study on binding energies of $\Lambda$ hypernuclei, Eur. Phys. J. Plus, 132, , 262271.

[9] Gal, A., et al., (2017) Strangeness in nuclear physics, Reviews of Modern Physics, 88(3), 201-212

[10] Bandō, H., Motoba, T., Žofka, J., (1990) Production, structure and decay of hypernuclei, International Journal of Modern Physics A, 05(21), 4021-4198.

[11] Samanta, C., et al., (2006) Mass Formula from Light to Hypernuclei, arXiv:nucl-th/0602063v. 